\title{
The Connes spectrum for actions of Abelian groups on continuous-trace algebras
}

\author{
STEVEN HURDER, DORTE OLESEN, IAIN RAEBURN \\ AND JONATHAN ROSENBERG
}

Department of Mathematics, University of Illinois at Chicago, P.O. Box 4348, Chicago, Illinois 60680, USA; Matematisk Institut, Universitetsparken 5, 2100 København $\varnothing$, Denmark; School of Mathematics, University of New South Wales, P.O. Box 1, Kensington, NSW 2033, Australia; Department of Mathematics, University of Maryland, College Park, Maryland 20742, USA.

(Received 26 August 1985 and revised 28 January 1986)

\begin{abstract}
We study the various notions of spectrum for an action $\alpha$ of a locally compact abelian group $G$ on a type $I C^{*}$-algebra $A$, and discuss how these are related to the structure of the crossed product $A \rtimes_{\alpha} G$. In the case where $A$ has continuous trace and the action of $G$ on $\hat{A}$ is minimal, we completely describe the ideal structure of the crossed product. A key role is played by the restriction of $\alpha$ to a certain 'symmetrizer subgroup' $S$ of the common stabilizer in $G$ of the points of $\hat{A}$. We show by example that, contrary to a conjecture of Bratteli, it is possble for $A \rtimes G$ to be primitive but not simple, provided that $S$ is not discrete. In such cases, the Connes spectrum $\Gamma(\alpha)$ differs from the strong Connes spectrum $\Gamma(\alpha)$ of Kishimoto. The counterexamples come from subtle phenomena in topological dynamics.
\end{abstract}

\section{0 . Introduction and preliminaries}

Let $(A, G, \alpha)$ be a $C^{*}$-dynamical system, i.e. a triple consisting of a $C^{*}$-algebra $A$, a locally compact group $G$ and a representation of $G$ as automorphisms of $A$ which are pointwise norm-continuous. In this paper we shall further assume that $A$ is of type $I$ and that $G$ is abelian. The structure of crossed products of such systems has been investigated in many papers over the past years under various more specific assumptions on the algebra or the action. (For surveys of the literature, see [P2], [Goot1], and [P1].) For the case where $A$ is commutative, hence of the form $C_{0}(X)$, a detailed knowledge of the structure of the primitive ideal space of the crossed product - in this context more often referred to as the transformation group $C^{*}$-algebra $C^{*}(G, X)$ - has been obtained by Williams in [Wi]. For the algebra $\mathscr{K}(\mathscr{H})$ of all compact operators on a Hilbert space $\mathscr{H}$, it is known that the primitive ideal space of the crossed product by an action $\alpha$ is always Hausdorff, in fact isomorphic to the quotient of the dual group $\hat{G}$ by the Connes spectrum: [Gr2] and [OI]. For more general type I algebras, results have been reached with additional assumptions on the action. In [PR2] it is shown that if the given action induces a 
trivial action on the primitive ideal space of $\boldsymbol{A}$, and the canonical action on each primitive quotient is implemented by a unitary group, then the primitive ideal space of the crossed product is mapped canonically onto the primitive ideal space of $A$, and for a rather large subclass of these actions, the locally unitary actions, Prim $\left(A \rtimes_{\alpha} G\right)$ becomes a locally trivial principal $\hat{G}$-bundle relative to the dual action of $\hat{G}$. For general actions on type I algebras the knowledge of $\operatorname{Prim}\left(A \rtimes_{\alpha} G\right)$ has, however, remained fragmentary. In this paper we show that there may be a good reason for this: the structure is complicated, and very general structure theorems cannot be expected. Indeed, as a starting point for any good structure theory one would want the crossed product to be simple under sufficiently strong assumptions on the action - but we shall give examples where the cross product is primitive but not simple.

At the 1977 Second US-Japan Conference Bratteli made the conjecture that the crossed product should be simple when $A$ was of type I, the action $G$-simple (i.e. the only $G$-invariant ideals are the trivial ones) and with full Connes spectrum. This was known to be true for $G$ discrete [OP1, 6.5], and subsequent to the conjecture was soon proved for $G$ compact or $A$ unital [OP.2, 3.11]. Bratteli restated the conjecture in $[\mathrm{Br}]$, and in the following years several special cases were settled in the affirmative: [ET], [OP3], [GO]. In the present paper, however, we show that in general the conjecture fails to be true.

In the case of a $G$-simple action on a type $I C^{*}$-algebra $A, A$ is necessarily liminal and homogeneous. If $A$ is unital or $G$ is compact, $A$ necessarily has continuous trace, but in the general case Larry Brown tells us that he has an example where $A$ is $G$-simple and liminal but has non-Hausdorff spectrum. Here, however, we shall show that even for a continuous-trace $C^{*}$-algebra $A$ with a $G$-simple action and full Connes spectrum, the crossed product may fail to be simple. The examples we exhibit arise in the cases of actions of $\mathbb{R} \times \mathbb{T}$ or $\mathbb{R} \times \mathbb{R}$ on algebras of the form $C_{0}(X, \mathscr{K}(\mathscr{H}))$, where $X$ is a second-countable locally compact Hausdorff space and $\mathscr{K}(\mathscr{H})$ is the algebra of all compact operators on some infinite dimensional (separable) Hilbert space.

Our way to these counterexamples to Bratteli's conjecture goes through a reduction of the original question to one in topological dynamics. We focus attention on a special subgroup of $G$, the symmetrizer group $S$ for the action. We show that for any $G$-simple action on a continuous trace algebra $A$, the primitive ideal space Prim $\left(A \rtimes_{\alpha} G\right)$ of the crossed product is homeomorphic to the quasi-orbit space for the associated action on Prim $\left(A \rtimes_{\alpha} S\right)$, thus extending a result in [RR, 2.1]. This sheds further light on the 'unwinding phenomenon' considered in [AM, Ch.I]. We then construct examples where the action on the Hausdorff space Prim $\left(A \rtimes_{\alpha} S\right)$ has more than one quasi-orbit, albeit the original action has full Connes spectrum. More precisely, we exhibit examples where there is one dense orbit on Prim $\left(A \rtimes_{\alpha} S\right)$, but not every orbit is dense.

\section{The symmetrizer group}

Let $A$ be a separable $C^{*}$-algebra of type I and let $G$ be a separable locally compact abelian group acting on $A$. Then the primitive ideal space Prim $A$ can be identified 
with $\hat{A}$, the space of unitary equivalence classes of irreducible representations of $A$, and $G$ acts in a natural way as a topological transformation group on this spectrum, which we shall henceforth denote by $X$. For $x$ in $X$, let

$$
G_{x}=\{t \in G \mid t x=x\}
$$

denote the isotropy group of $x$, and let

$$
G x^{-}=\left\{y \in X \mid G y^{-}=G x^{-}\right\}
$$

denote the quasi-orbit of $x$, i.e. the points in the spectrum with the same $G$-orbit closure as $x$. For every $y$ in $G x^{\sim}, G_{y}=G_{x}$ by [Goot2, lemma 2.2] combined with [GO, proposition 2.2], and all isotropy groups are necessarily closed. Denoting the orbit space by $X / G$, we use the symbol $2(X / G)$ for the quasi-orbit space, which is given the quotient topology.

Now each $x$ in $X$ determines a multiplier representation $u$ with multiplier $\omega_{x}$ which implements the action of $G_{x}$ :

$$
u_{s} \pi_{x}(a) u_{s}^{*}=\pi_{x}\left(\alpha_{s}(a)\right) \quad \forall a \in A, s \in G_{x},
$$

and $\omega_{x}$ determines a subgroup

$$
S_{x}=\left\{s \in G_{x} \mid \omega_{x}(s, t)=\omega_{x}(t, s) \quad \forall t \in G_{x}\right\} .
$$

The relevance of $S_{x}$ for the ideal structure of $A \rtimes_{\alpha} G$ originates in the fact that if $\omega_{x}$ is totally skew, i.e. $S_{x}$ is trivial, all irreducible $\omega_{x}$-representations are weakly equivalent - [BK], [Gr2]. Its role has slowly attracted more attention (cf. [ET], [GO] and [Goot1] \& [Goot2]), and we shall continue these investigations in the present paper. At this point it seems suitable to search for a name for $S_{x}$, and we propose to call it the symmetrizer group of $x$.

Recall the following crucial result from [Goot2, theorem 2.3]:

TheOREM 1.0. Let $(A, G, \alpha)$ be a separable $C^{*}$-dynamical system with $G$ locally compact abelian and $A$ of type I. Let $x$ and $y$ have the same quasi-orbit. Then $\omega_{x}$ and $\omega_{y}$ determine the same cocycle class, and $S_{x}=S_{y}$.

Note that the second statement of the theorem follows from the first, as $S_{x}$ clearly only depends on the cohomology class of $\omega_{x}$. By the Mackey obstruction associated to $x$ in $X$ we mean the cocycle class of $\omega_{x}$, and the above implies that this is constant on each quasi-orbit. We note that the common symmetrizer group $S$ on a given quasi-orbit $G x^{\sim}$ is the smallest closed subgroup of $G_{x}$ such that the Mackey obstruction is trivial on $S$ and totally skew on $G_{x} / S$.

Recall from [Gr2, proposition 9] that there are a number of maps from the ideal space of a crossed product to that of a crossed product by a subgroup, and vice versa. In what follows, we shall be concerned with the following two maps between the ideal space $I\left(A \times_{\alpha} H\right)$ of a crossed product by some closed subgroup $H$ of $G$ and the ideal space $\mathscr{I}\left(A \rtimes_{\alpha} S\right)$ of the crossed product by a smaller closed subgroup $S \subseteq H$. In the formulations, we are viewing $A \rtimes_{\alpha} S$ as a subalgebra of the multiplier algebra $M\left(A \rtimes_{\alpha} H\right)$ : 
(1) The restriction map from $\mathscr{I}\left(A \rtimes_{\alpha} H\right)$ into $\mathscr{I}\left(A \rtimes_{\alpha} S\right)$ which takes

$$
I \mapsto \operatorname{Res}_{S}^{H} I=\left\{y \in A \rtimes_{\alpha} S \mid x y, y x \in I \quad \forall x \in A \rtimes_{\alpha} H\right\}
$$

and

(2) The extension map from $\mathscr{I}\left(A \rtimes_{\alpha} S\right)$ into $\mathscr{I}\left(A \rtimes_{\alpha} H\right)$ given by

$$
J \mapsto \operatorname{Ex}_{S}^{H} J=\text { the ideal generated by }\left\{y d \mid y \in J, d \in A \rtimes_{\alpha} H\right\} .
$$

Note that when $(\pi, U)$ is a covariant representation of $(A, H, \alpha)$,

$$
\operatorname{Res}_{S}^{H}(\operatorname{ker}(\pi \times U))=\operatorname{ker}\left(\pi \times\left. U\right|_{S}\right)
$$

by [Gr2, proposition 9(ii)]. Also, in what follows, we shall freely use the isomorphism $A \rtimes_{\alpha} H \cong\left(A \rtimes_{\alpha} S\right) \rtimes^{\tau} H$ of [Gr2, proposition 1], where $\rtimes^{\tau}$ stands for the twisted crossed product.

When $A \rtimes_{\alpha} S$ is the crossed product by a subgroup of $H, H$ itself acts canonically on the dense subalgebra $C_{c}(S, A)$ of continuous functions with compact support from $S$ into $A$ by

$$
\left(\alpha_{h} y\right)(s)=\alpha_{h}(y(s)), \quad y \in C_{c}(S, A),
$$

and this action extends to all of $A \rtimes_{\alpha} S$. In turn, it induces an action on the ideal space $\mathscr{I}\left(A \rtimes_{\alpha} S\right)$, and on the $H$-invariant ideals in $A \rtimes_{\alpha} S$, the extension map Ex ${ }_{S}^{H}$ coincides with the induction map $\operatorname{Ind}_{S}^{H}$ by [Gr2, proposition 13(ii)]. This allows us to use the extension map in the proof of the following theorem:

Theorem 1.1. Let $(A, G, \alpha)$ be a separable $C^{*}$-dynamical system with $A$ continuoustrace and $G$ locally compact abelian. Suppose that the isotropy group $H$ is the same for every point $x$ in the spectrum $X$ of $A$, and that the symmetrizer group $S$ is also constant on $X$. Then $\operatorname{Res}_{S}^{H}$ induces a homeomorphism of the primitive ideal space Prim $\left(A \rtimes_{\alpha} H\right)$ onto Prim $\left(A \rtimes_{\alpha} S\right)$, with inverse $\operatorname{Ind}_{S}^{H}$, and this homeomorphism is G-equivariant.

Proof. Note that $H$ acts trivially on $X$, hence preserves the fibres of the projection Prim $\left(A \rtimes_{\alpha} S\right) \rightarrow X$. Since $H$ is abelian, $H$ also acts trivially on $S$, and so acts trivially on Prim $\left(A \rtimes_{\alpha} S\right)$. Then restriction maps Prim $\left(A \rtimes_{\alpha} H\right)$ into Prim $\left(A \rtimes_{\alpha} S\right)$, since a primitive ideal $J$ in $A \rtimes_{\alpha} H$ by [Gr2, proposition 11(i)] maps onto an $H$-invariant ideal $\operatorname{Res}_{S}^{H} J$, which by quasi-regularity uniquely determines an $H$-quasi-orbit in Prim $\left(A \rtimes_{\alpha} S\right)$ - but the quasi-orbits are just single points.

Let $I \in \operatorname{Prim}\left(A \rtimes_{\alpha} S\right)$, then by [Gr2, proposition 12(ii)],

$$
\left(A \rtimes_{\alpha} H\right) / \operatorname{Ex}_{S}^{H} I=\left(\left(A \rtimes_{\alpha} S\right) / I\right) \rtimes^{\tau} H,
$$

where the right hand side denotes the twisted crossed product of the quotient algebra $\left(A \rtimes_{\alpha} S\right) / I$ by the canonical action of $H$. Now since we know that $A \rtimes_{\alpha} S$ is liminal by [PR2, proposition 2.1], every primitive quotient of $A \rtimes_{\alpha} S$ is isomorphic to the compact operators $\mathscr{K}(\mathscr{H})$ on some Hilbert space $\mathscr{H}$. Furthermore, any irreducible representation $\rho: A \rtimes_{\alpha} S \rightarrow \mathscr{K}(\mathscr{H})$ is of the form $\rho=\pi \times \lambda[\mathrm{P1}, 7.6 .4]$, where $(\pi, \lambda, \mathscr{H})$ is a covariant representation of $(A, S, \alpha \mid S)$. By [PR2, proposition 2.1] $\pi$ is itself an irreducible representation of $A$, hence the unitary cocycle implementing the action of $H$ on $\mathscr{K}(\mathscr{H})$ is totally skew on $H / S$. It then follows from [Gr2, proposition 32] that the twisted crossed product in $(*)$ is simple. This implies that $\operatorname{Ex}_{S}^{H} I$ is a maximal ideal, a fortiori primitive, so the extension map takes primitive ideals to primitive 
ideals. Furthermore, the general inclusion $\operatorname{Ex}_{S}^{H} \operatorname{Res}_{S}^{H} J \subseteq J$ becomes equality on account of the maximality, and combined with the equality

$$
\operatorname{Res}_{S}^{H} \operatorname{Ex}_{S}^{H} I=\operatorname{Res}_{S}^{H} \operatorname{Ind}_{S}^{H} I=I
$$

[Gr2, proposition 11], this proves that we have a bijection between the primitive ideal spaces in question. The continuity of both maps now ensures we have a homeomorphism. The $G$-equivariance follows from a simple calculation:

$$
\begin{aligned}
t \cdot\left(\operatorname{Res}_{S}^{H}(\operatorname{ker}(\pi \times U))\right) & =t \cdot \operatorname{ker}\left(\pi \times\left. U\right|_{S}\right) \\
& =\operatorname{ker}\left(t \cdot \pi \times\left. U\right|_{S}\right) \\
& =\operatorname{Res}_{S}^{H}(\operatorname{ker}(t \cdot \pi \times U)) \\
& =\operatorname{Res}_{S}^{H}(t \cdot \operatorname{ker}(\pi \times U)),
\end{aligned}
$$

for $t \in G, \pi \times U$ an irreducible covariant representation of $(A, H)$.

Proposition 1.2. Let $(A, G, \alpha), H$ and $S$ be as in theorem 1.1. Then Prim $\left(A \rtimes_{\alpha} G\right)$ is homeomorphic to the quasi-orbit space $2\left(\left(A \rtimes_{\alpha} S\right) \hat{\gamma} G\right)$, and $\operatorname{Prim}\left(A \rtimes_{\alpha} S\right)$ is homeomorphic to $\left(A \rtimes_{\alpha} S\right)^{\hat{}}$.

Proof. By the positive solution of the generalized Effros-Hahn conjecture [GR, 3.1 and 3.2], every primitive ideal of $A \rtimes_{\alpha} G$ is induced from $(A, H)$. So using $E H$ regularity and [Gr2, theorem 24] as in the second paragraph of the proof of [RR, proposition 2.1], we see that $\operatorname{Prim}\left(A \rtimes_{\alpha} G\right)$ is homeomorphic to the space $2\left(\operatorname{Prim}\left(A \rtimes_{\alpha} H\right) / G\right)$. Now by theorem 1.1 , Prim $\left(A \rtimes_{\alpha} H\right)$ is $G$-equivariantly homeomorphic to Prim $\left(A \rtimes_{\alpha} S\right)$, which in turn may be identified with $\left(A \rtimes_{\alpha} S\right)^{\wedge}$ since this algebra is type $\mathrm{I}$. Hence

$$
\mathscr{2}\left(\operatorname{Prim}\left(A \rtimes_{\alpha} H\right) / G\right) \cong \mathscr{2}\left(\left(A \rtimes_{\alpha} S\right)^{\wedge} / G\right) .
$$

Remark. If $S$ is compactly generated, then since the action of $S$ on $A$ is pointwise unitary, it is locally unitary by [R, corollary 2.2]. This implies by [PR2, proposition 4.1] that $A \rtimes_{\alpha} S$ has continuous trace and that $\left(A \rtimes_{\alpha} S\right)^{\wedge} \rightarrow X$ is a locally trivial principal $\hat{S}$-bundle.

Proposition 1.3. Let $(A, G, \alpha)$ be a $C^{*}$-dynamical system with $A$ continuous-trace and $G$ locally compact abelian. Assume that the action is $G$-simple, i.e. has no non-trivial $G$-invariant ideals. Then there is one fixed isotropy group $H$ for the action on the spectrum of $A$, and one fixed symmetrizer group $S$, and $\operatorname{Prim}\left(A \rtimes_{\alpha} G\right)$ is homeomorphic to the quasi-orbit space $\mathscr{2}\left(\left(A \rtimes_{\alpha} S\right) \hat{\gamma} / G\right)$.

Proof. Since the action on the spectrum $X$ of $A$ is minimal, the uniqueness of $H$ and $S$ follows immediately from their constancy on quasi-orbits. The conclusion about Prim $\left(A \rtimes_{\alpha} G\right)$ is then an immediate corollary of 1.2.

Corollary 1.4. Let $(A, G, \alpha)$ be a G-simple $C^{*}$-dynamical system, where $A$ has continuous trace and $G$ is locally compact abelian. Then, $S$ denoting the common symmetrizer group,

(i) $A \rtimes_{\alpha} G$ is simple if and only if every orbit for the G-action on Prim $\left(A \rtimes_{\alpha} S\right)$ is dense; and

(ii) $A \rtimes_{\alpha} G$ is prime if and only if there is at least one dense orbit for the G-action on $\operatorname{Prim}\left(A \rtimes_{\alpha} S\right)$. 
Proof. This follows from proposition 1.3 after observing that:

(i) $A \rtimes_{\alpha} G$ is simple if and only if Prim $\left(A \rtimes_{\alpha} G\right)$ consists of just one point, hence if and only if $\mathscr{Q}\left(\left(A \rtimes_{\alpha} S\right) \wedge / G\right)$ consists of just one point,

(ii) $A \rtimes_{\alpha} G$ is prime if and only if 0 is a primitive ideal, hence if and only if at least one orbit closure for the action on Prim $\left(A \rtimes_{\alpha} S\right)$ is all of Prim $\left(A \rtimes_{\alpha} S\right)$.

2. Various spectra and their actions on $\left(A \rtimes_{\alpha} S\right)^{\wedge}$

Let $(A, G, \alpha)$ be a separable $C^{*}$-dynamical system with $G$ locally compact abelian, and let $\hat{G}$ denote the dual group of $G$. Recall that for any open subset $U$ of $\hat{G}$, the spectral subspace $A^{\alpha}(U)$ is defined to be the closed linear span of elements of the form

$$
\alpha_{f}(a)=\int_{G} f(t) \alpha_{t}(a) d t,
$$

where $f \in L^{1}(G)$ and $\hat{f}$ has compact support in $U$.

An element $\gamma$ in $\hat{G}$ is said to belong to the Arveson spectrum $\operatorname{Sp}(\alpha)$ of $(A, G, \alpha)$ if

$$
A^{\alpha}(U) \neq\{0\}
$$

for every neighbourhood $U$ of $\gamma$, and to belong to the Connes spectrum $\Gamma(\alpha)$ if it belongs to $\operatorname{Sp}(\alpha \mid B)$ for every non-zero $G$-invariant hereditary $C^{*}$-subalgebra $B$ of $A$.

Using the dual $C^{*}$-dynamical system $\left(A \rtimes_{\alpha} G, \hat{G}, \hat{\alpha}\right)$, the Connes spectrum can be characterized as follows [OP1, 5.4]: an element $\gamma$ in $\hat{G}$ belongs to $\Gamma(\alpha)$ if and only if $I \cap \hat{\alpha}_{\gamma}(I)$ is non-zero for every non-zero closed ideal $I$ of $A \rtimes_{\alpha} G$.

It is immediately apparent from this way of looking at the Connes spectrum that it must be a (closed) subgroup of $\hat{G}$ - a property that often eases its computation. This is in marked contrast to $\operatorname{Sp}(\alpha)$, which may be any closed subset of $\hat{G}$, and is most easily thought of as the 'spectrum of the generator' of the group action (in fact a precise statement for the cases $G=\mathbb{Z}$ or $G=\mathbb{R}$, in the latter case taking the infinitesimal generator). In [Ki], Kishimoto introduced variations of these spectra. He defined the strong Arveson spectrum $\widetilde{\mathrm{Sp}}(\alpha)$ to be the set of $\gamma$ in $\hat{G}$ for which the smallest hereditary $C^{*}$-subalgebra $A(U)$ generated by the spectral subspace $A^{\alpha}(U)$ was all of $A$ for any neighbourhood $U$ of $\gamma$. He could then show that $\widetilde{\mathrm{Sp}}(\alpha)$ is always a closed subsemigroup of $\hat{G}$, and this property is in turn inherited by the strong Connes spectrum, $\tilde{\Gamma}(\alpha)$, defined to be the intersection of all $\widetilde{\mathrm{Sp}}(\alpha \mid B), B$ running through the non-zero $G$-invariant hereditary $C^{*}$-subalgebras of $A$.

In [Ki, 3.4], Kishimoto obtained the following characterization of the strong Connes spectrum: an element $\gamma$ in $\hat{G}$ belongs to $\tilde{\Gamma}(\alpha)$ if and only if $\hat{\alpha}_{\gamma}(I) \subseteq I$ for every ideal $I$ of $A \rtimes_{\alpha} G$.

This characterization clearly exhibits the semigroup property of $\tilde{\Gamma}(\alpha)$, and also makes it clear that one cannot in general expect $\tilde{\Gamma}(\alpha)$ to be a subgroup like $\Gamma(\alpha)$. In fact, $[\mathrm{Ki}, 5.2]$ exhibits an example where $\tilde{\Gamma}(\alpha)=\mathbb{Z}_{+}$. For a number of special cases it is, however, possible to prove that $\tilde{\Gamma}(\alpha)$ is indeed a subgroup, and this is desirable as it facilitates the computation of this otherwise evasive invariant. Obviously, when 
$\tilde{\Gamma}(\alpha)$ is known to be a group, the above characterization simplifies - then $\gamma \in \tilde{\Gamma}(\alpha)$ if and only if $\hat{\alpha}_{\gamma}(I)=I$ for every ideal $I$ of $A \rtimes_{\alpha} G$. In fact, defining

$$
\tilde{\tilde{\Gamma}}(\alpha)=\left\{\gamma \in \hat{G} \mid \hat{\alpha}_{\gamma}(I)=I \text { for every ideal } I \text { of } A \rtimes_{\alpha} G\right\}
$$

we have another variation of the Connes spectrum, which is clearly a closed subgroup of the regular Connes spectrum $\Gamma(\alpha)$. Note that

$$
\tilde{\tilde{\Gamma}}(\alpha) \subseteq \tilde{\Gamma}(\alpha) \subseteq \Gamma(\alpha) \text {. }
$$

In the example mentioned above where $\tilde{\Gamma}(\alpha)=\mathbb{Z}_{+}$, the $C^{*}$-algebra under consideration is $O_{\infty}$, the Cuntz algebra generated by a sequence of isometries. This $C^{*}$-algebra is highly non-type I, and one might start wondering whether the two spectral notions $\tilde{\Gamma}(\alpha)$ and $\tilde{\Gamma}(\alpha)$ might coincide in the type I case. Indeed, it is immediate that if $A=C_{0}(X)$, then both $\widetilde{\mathrm{Sp}}(\alpha)$ and $\tilde{\Gamma}(\alpha)$ are necessarily subgroups, hence $\tilde{\Gamma}(\alpha)=$ $\tilde{\Gamma}(\alpha)$ - in fact in this case it is possible to prove even more:

$$
\tilde{\tilde{\Gamma}}(\alpha)=\tilde{\Gamma}(\alpha)=\widetilde{\mathrm{Sp}}(\alpha)=\bigcap_{x \in X} G_{x}^{\perp},
$$

where $G_{x}^{\perp}$ denotes the annihilator of the isotropy group $G_{x}$. It is also true that when $G$ is discrete, $\tilde{\Gamma}(\alpha)$ is necessarily a subgroup (also in the non-type I case), being a closed subsemigroup of a compact group [Wr], and we can further show that the following holds:

Proposition 2.0. Let $(A, G, \alpha)$ be a $C^{*}$-dynamical system where $A \rtimes_{\alpha} G$ is of type I. Then $\tilde{\Gamma}(\alpha)=\tilde{\Gamma}(\alpha)$. This is especially true whenever $A$ is type $\mathrm{I}$ and $G$ is compact.

Proof. When $A \rtimes_{\alpha} G$ is of type I, we know from [La, theorem 1] that an automorphism which maps every ideal into itself is necessarily universally weakly inner, hence maps every ideal onto itself. Thus $\tilde{\Gamma}(\alpha)=\tilde{\Gamma}(\alpha)$.

That $A \rtimes_{\alpha} G$ is of type $I$ when $A$ is of type $I$ and $G$ is compact is an easy application of Takai duality, for the abelian group case considered here. Indeed, the double crossed product is by Takai duality $A \otimes \mathscr{K}\left(L^{2}(G)\right.$ ), hence of type $\mathrm{I}$, and the crossed product is a $C^{*}$-subalgebra of this.

In $\S 4$ of this paper, we shall see that one can go somewhat further along these lines in different special cases where more assumptions are being put on the action.

At this point, however, we want to turn to the question of the action of these spectra on $\mathscr{I}\left(A \rtimes_{\alpha} S\right)$. Recall that the dual action is given on the dense subalgebra $C_{c}(G, A)$ of continuous functions with compact support from $G$ into $A$ simply by

$$
\left(\hat{\alpha}_{\gamma} y\right)(t)=(t, \gamma) y(t) \text {, }
$$

i.e. by pointwise multiplication by a character, and this action clearly extends to $A \rtimes_{\alpha} G$ and even further to the multiplier algebra $M\left(A \rtimes_{\alpha} G\right)$. Now the crossed product by a subgroup can naturally be viewed as embedded in $M\left(A \rtimes_{\alpha} G\right)$, and can be characterized inside of this $C^{*}$-algebra as a certain $C^{*}$-subalgebra of the fixed-points under the dual action restricted to the annihilator of the subgroup in question [LOP, theorem 2.1]. In particular, taking here the subgroup to be $S$, the dual action on $A \rtimes_{\alpha} S$ can be viewed both as the dual action on the crossed product by the group $\hat{S}$, or as the canonical restriction of the dual action from $M\left(A \rtimes_{\alpha} G\right)$, 
the point being that $\hat{S}$ is isomorphic to the quotient group $\hat{G} / S^{\perp}$, and that $S^{\perp}$ leaves the elements of $A \rtimes_{\alpha} S$ pointwise fixed.

Looking at a $G$-simple system, we have as before a fixed isotropy group $H$ and a fixed symmetrizer group $S$, and a homeomorphism from Prim $\left(A \rtimes_{\alpha} G\right)$ to $\mathscr{Q}\left(\left(A \rtimes_{\alpha} S\right) \hat{~} / G\right)$. As described above, the actions of the spectra on $\mathscr{I}\left(A \rtimes_{\alpha} G\right)$ are well-known, and this now enables us to deduce results about the actions on $\left(A \rtimes_{\alpha} S\right)^{\wedge}$, as indeed we have the following observation:

Lemma 2.1. Let $(A, G, \alpha)$ be a $G$-simple $C^{*}$-dynamical system where $A$ is of type I and $G$ is locally compact abelian. Then the common symmetrizer group $S$ satisfies $S^{\perp} \subseteq \tilde{\tilde{\Gamma}}(\alpha)$.

Proof. By the positive solution of the generalized Effros-Hahn conjecture [GR, 3.1 \& 2], every primitive ideal $J$ of $A \rtimes_{\alpha} G$ is induced from an ideal of $A \rtimes_{\alpha} H$, and this can be chosen $G$-invariant by [Gr2, proposition 11(ii)]. Applying our reasoning in the proof of theorem 1.1 we see that this ideal $J^{\prime}$ in $A \rtimes_{\alpha} H$ is induced from an ideal $I$ in $A \rtimes_{\alpha} S$ which is also $G$-invariant. Thus $J$ is induced from a $G$-invariant ideal of $A \rtimes_{\alpha} S$, or, by [Gr2, proposition 13(ii)], is an extension of such an ideal, and hence we immediately see from the definition of the extension map that $J$ is $S^{\perp}$-invariant.

We note that in fact the above lemma could be derived from the proof of [GO, 2.6], where it is shown that in a situation with (possibly) varying symmetrizer groups for the action on $X=\operatorname{Prim} A$, the intersection $\bigcap_{x \in X} S_{x}^{\perp}$ is always contained in $\Gamma(\alpha)$. The above simple proof is included for the benefit of the reader.

We are now in a position to point out that for actions on type I algebras, the Connes spectra are effectively acting only on the ideals of $A \rtimes_{\alpha} S$, as $S^{\perp}$ leaves all ideals of the 'big' crossed product $A \rtimes_{\alpha} G$ invariant. In fact it is the action on the $G$-invariant ideals ${ }^{G} \mathscr{I}\left(A \rtimes_{\alpha} S\right)$ of $A \rtimes_{\alpha} S$ - or the quasi-orbits in the primitive ideal space of $A \rtimes_{\alpha} S$ - that determines the relation with the original action on $A$. Let us first transcribe the characterization of the $\Gamma$-spectra to $A \rtimes_{\alpha} S$, viewing the dual action on this as a quotient action of the one on $A \rtimes_{\alpha} G$ :

Proposition 2.2. Let $(A, G, \alpha)$ be a G-simple $C^{*}$-dynamical system where $A$ is of type I and $G$ is locally compact abelian. Denote by $q$ the quotient map from $\hat{G}$ to $\hat{S}$. Then:

(i) $\tilde{\tilde{\Gamma}}(\alpha)$ is the inverse image under $q$ of the elements $q(\gamma)$ in $\hat{S}$ that leave every $G$-invariant ideal of $A \rtimes_{\alpha} S$ invariant;

(ii) $\tilde{\Gamma}(\alpha)$ is the inverse image under q of the elements $q(\gamma)$ in $\hat{S}$ for which $\hat{\alpha}_{q(\gamma)}(I) \subseteq I$ for every $I$ in ${ }^{G} \mathscr{I}\left(A \rtimes_{\alpha} S\right)$; and

(iii) $\Gamma(\alpha)$ is the inverse image under $q$ of the elements $q(\gamma)$ in $\hat{S}$ such that $\hat{\alpha}_{q(\gamma)}(I) \cap$ $I \neq 0$ for every non-zero $G$-invariant ideal $I$ of $A \rtimes_{\alpha} S$.

Proof. First note that what we were saying in the proof of lemma 2.1 was that every primitive ideal of $A \rtimes_{\alpha} G$ is in fact induced from a $G$-invariant ideal in $A \rtimes_{\alpha} S$. Since every ideal in $A \rtimes_{\alpha} G$ is an intersection of primitive ideals, and induction preserves intersection, this implies that every ideal in $A \rtimes_{\alpha} G$ is induced. Furthermore, the dual action respects the induction in the sense that $\hat{\alpha}_{\gamma}\left(\operatorname{Ind}_{S}^{G} I\right)=$ 
$\operatorname{Ind}_{S}^{G}\left(\hat{\alpha}_{q(\gamma)} I\right)$, where we confuse the notation for the dual actions on $A \rtimes_{\alpha} G$ and $A \rtimes_{\alpha} S$.

(i) Let $\gamma \in \tilde{\tilde{\Gamma}}(\alpha)$, and let $I$ be a $G$-invariant ideal in $A \rtimes_{\alpha} S$. Then

$$
\operatorname{Ind}_{S}^{G}\left(\hat{\alpha}_{q(\gamma)} I\right)=\hat{\alpha}_{\gamma}\left(\operatorname{Ind}_{S}^{G} I\right)=\operatorname{Ind}_{s}^{G} I \text {, }
$$

and by injectivity of Ind on the $G$-invariant ideals, $\hat{\alpha}_{q(\gamma)} I=I$. Conversely, if we have $\eta$ in $\hat{S}$, then choosing $\gamma$ in $\hat{G}$ with $q(\gamma)=\eta$, we see that for a given ideal $J$ in $A \rtimes_{\alpha} G$, there is a $G$-invariant ideal $I$ in $A \rtimes_{\alpha} S$ such that $J=\operatorname{Ind}_{S}^{G} I$, and supposing now that $\hat{\alpha}_{\eta}(I)=I$ we get

$$
\hat{\alpha}_{\gamma}(J)=\hat{\alpha}_{\gamma}\left(\operatorname{Ind}_{S}^{G} I\right)=\operatorname{Ind}_{S}^{G}\left(\hat{\alpha}_{q(\gamma)} I\right)=\operatorname{Ind}_{S}^{G} I=J .
$$

The proofs of (ii) and (iii) are verbatim transcriptions of this line of argument.

That the role of the different $\Gamma$-spectra is to determine the relation between the $G$-invariant ideals in $A \rtimes_{\alpha} S$ and the ones in our original algebra $A$ now becomes very clear:

Theorem 2.3. Let $(A, G, \alpha)$ be a $C^{*}$-dynamical system with $A$ of type $\mathrm{I}$, common symmetrizer group $S$ and common isotropy group $H$ in the locally compact abelian group $G$. Then:

(i) $A \rtimes_{\alpha} S$ is G-simple if and only if $\tilde{\Gamma}(\alpha)=\hat{G}$ and $A$ is G-simple;

(ii) $A \rtimes_{\alpha} S$ is G-prime if and only if $\Gamma(\alpha)=\hat{G}$ and $A$ is $G$-prime.

Proof. (i) Assume that $A \rtimes_{\alpha} S$ is $G$-simple; then by proposition 2.2(i) $\tilde{\Gamma}(\alpha)=\hat{G}$, and a fortiori $\tilde{\Gamma}(\alpha)=\hat{G}$. Since every ideal $I$ in $A$ is $S$-invariant, $I \rtimes_{\alpha} S$ is a non-trivial ideal whenever $I$ is non-trivial, hence $G$-simplicity of $A$ follows from that of $A \rtimes_{\alpha} S$. Conversely, if $\tilde{\Gamma}(\alpha)=\hat{G}$ and $A$ is $G$-simple, then $\tilde{\tilde{\Gamma}}(\alpha)=\hat{G}$ since $\tilde{\Gamma}(\alpha)=\hat{G}$ implies that it is a group, and so every $G$-invariant ideal of $A \rtimes_{\alpha} S$ is also $\hat{S}$-invariant, hence maps onto an ideal of $A$ which is non-trivial if it is itself non-trivial, [Ol, 2.1]. (Note that the map $I$ as defined in [OI] coincides with restriction on $S$-invariant ideals.) Thus the $G$-simplicity of $A$ implies $G$-simplicity of $A \rtimes_{\alpha} S$.

(ii) Assume that $A \rtimes_{\alpha} S$ is $G$-prime; then by proposition 2.2 (iii) we have $\Gamma(\alpha)=\hat{G}$, and whenever $I_{1}$ and $I_{2}$ are non-zero $G$-invariant ideals of $A$, they induce to non-zero $G$-invariant ideals with non-zero intersection. Now recalling that induction preserves intersection, we see that $I_{1} \cap I_{2}$ induces to a non-zero ideal, hence is non-zero. Conversely, assume that $A$ is $G$-prime and $\Gamma(\alpha)=\hat{G}$. Then taking $J_{1}$ and $J_{2}$ to be $G$-invariant ideals of $A \rtimes_{\alpha} S$, we can find some $\eta$ in $\hat{S}$ such that $J_{1} \cap \hat{\alpha}_{\eta}\left(J_{2}\right) \neq$ 0 - indeed, if not, the ideal spanned by $\left\{\hat{\alpha}_{\xi}\left(J_{2}\right), \xi \in \hat{S}\right\}$ would be an $\hat{S}$-invariant ideal $J$ such that $J_{1} \cap J=0$, and the ideal $L$ spanned by $\left\{\hat{\alpha}_{\xi}\left(J_{1}\right)\right\}$ would be an $\hat{S}$-invariant ideal such that $L \cap J=0$. But then, reasoning as above, the $\hat{S}$-invariance of $L$ and $J$ would imply the existence of non-zero ideals $L^{\prime}$ and $J^{\prime}$ in $A$ that induced to $L$ and $J$, and the $G$-invariance of $J_{1}$ and $J_{2}$ ensures $G$-invariance of $L$ and $J$, hence of $L^{\prime}$ and $J^{\prime}$, thus $L^{\prime} \cap J^{\prime}=0$, a contradiction. Once $J_{1} \cap \hat{\alpha}_{\eta}\left(J_{2}\right) \neq 0$, then since $\Gamma(\alpha)=\hat{G}$, we have by proposition $2.2($ iii)

$$
\hat{\alpha}_{\eta^{-1}}\left(J_{1} \cap \hat{\alpha}_{\eta}\left(J_{2}\right)\right) \cap J_{1} \cap \hat{\alpha}_{\eta}\left(J_{2}\right) \neq 0,
$$

hence $J_{1} \cap J_{2} \neq 0$, and $A \rtimes_{\alpha} S$ is $G$-prime. 
Putting together theorem 1.1 and theorem 2.3, we have now rederived the well-known results ([Ki, theorem 3.5] \& [OP1, theorem 5.8]) that:

(ii) $A \rtimes_{\alpha} G$ is simple if and only if $\tilde{\Gamma}(\alpha)=\hat{G}$ and $A$ is $G$-simple; and

(ii) $A \rtimes_{\alpha} G$ is prime if and only if $\Gamma(\alpha)=\hat{G}$ and $A$ is $G$-prime.

Our reason for writing this out in detail is that it elucidates the role of the symmetrizer group and clarifies what the various $\Gamma$-spectra do. That simplicity of $A \rtimes_{\alpha} G$ could be deduced from $G$-simplicity of $A \rtimes_{\alpha} S$ was earlier observed in [Goot1, p. 317].

One very good reason for focusing on the actions on the ideals of $A \rtimes_{\alpha} S$, or, more precisely, on the $G$-quasi-orbits in Prim $\left(A \rtimes_{\alpha} S\right)$, is that the structure of this crossed product is much simpler than that of the crossed product by the entire group $G$. In the ensuing section we shall see how the machinery developed in [PR2], [R], and $[\mathbf{R R}]$ to study such 'small' corssed products can be used to good advantage.

\section{Realization of cocycles by group actions on $C^{*}$-algebras}

We saw in the previous sections that if a (second-countable) locally compact abelian group $G$ acts on a (separable) continuous-trace algebra $A$ with $\hat{A}=X$, and if $G$ acts minimally on $X$ (i.e. $A$ is $G$-simple), then the ideal structure of the crossed product $A \rtimes G$ is determined by the topological dynamics of the action of $G$ on $(A \rtimes S)^{\wedge}$. As above, we denote by $H$ the common isotropy group in $G$ of all points $x \in X$, and by $S$ the smallest closed subgroup of $H$ such that the Mackey obstruction is trivial on $S$ and totally skew on $H / S$. Our aim now is to construct examples where $A \rtimes G$ is primitive but not simple, and more generally, to study the possibilities for the crossed product and their relation to the Connes spectrum $\Gamma(\alpha)$. To do this, we use the above theory to reduce the problem to a topological one. But to know that topological counterexamples will give $C^{*}$-algebraic counterexamples, we need some 'cocycle realization theorems.' These are the subject of the present section.

We begin by recalling some of the theory developed in [PR1], [PR2], [R] and [RR]. For simplicity we take $A=C_{0}(X, \mathscr{K})$, where $X$ is a second-countable locally compact space and $\mathscr{K}$ is the algebra of compact operators on an infinite-dimensional separable Hilbert space, though much of the theory applies to more general continuous-trace algebras. Then

$$
\text { Aut } A \cong\left(\text { Aut }_{C_{0}(X)} A\right) \rtimes \text { Homeo }(X)
$$

is the semidirect product of the spectrum-fixing automorphisms by the homeomorphism group of $X$, and there is an exact sequence

$$
1 \rightarrow \operatorname{Inn} A \rightarrow \text { Aut }_{C_{0}(X)} A \rightarrow H^{2}(X, \mathbb{Z}) \rightarrow 1
$$

which is topological if the Čech group $H^{2}(X, \mathbb{Z})$ is countable (in particular, if $X$ is compact) ([PR1]; see also [RR, theorems 0.5 and 0.8$]$ ). Suppose $G$ is a secondcountable locally compact group (for the moment, it need not be abelian) and $\tau$ is an action of $G$ on $X$ by homeomorphisms, identified with an action of $G$ on $C_{0}(X)$. Then we obtain an action $\tau \otimes$ id of $G$ on $C_{0}(X) \otimes \mathscr{K} \cong A$, and if $\alpha$ is any other action of $G$ on $A$ inducing $\tau$ on $X$, then

$$
t \mapsto \alpha_{t}\left(\tau_{t} \otimes \mathrm{id}\right)^{-1}
$$


is a continuous 1 -cocycle

$$
\varphi: G \rightarrow \text { Aut }_{C_{0}(X)} A .
$$

If $G$ is connected and $H^{2}(X, \mathbb{Z})$ is countable, or if $H^{2}(X, \mathbb{Z})=0$, then $\varphi$ must take values in Inn $A$ and defines via the exact sequence

$$
H^{1}(G, U(M(A))) \rightarrow H^{1}(G, \operatorname{Inn} A) \stackrel{\partial}{\rightarrow} H^{2}(G, C(X, \mathbb{T}))
$$

a class

$$
c(\alpha)=\partial([\varphi]) \in H^{2}(G, C(X, \mathbb{T})),
$$

the vanishing of which is the unique obstruction to exterior equivalence of $\alpha$ with $\tau \otimes$ id [RR, corollary 0.13 ]. (Here we use C. C. Moore's cohomology theory based on Borel cochains $[\mathbf{M}]$ and $C(X, \mathbb{T})$ is given the Polish topology of uniform convergence on compacta in $X$.) Since the isomorphism class of the crossed product $A \rtimes_{\alpha} G$ (even as a $\hat{G}$-algebra, if $G$ is abelian) only depends on the exterior equivalence class of $\alpha$ (see e.g. [P2, p. 277] and [RR, theorem 0.10] for more precise statements), the cohomology class $c(\alpha)$ is an important invariant of the crossed product, and we need to see which classes in $H^{2}(G, C(X, \pi))$ can arise. Our first realization theorem says that all classes arise.

Proposition 3.1. Let $X$ be a second-countable locally compact Hausdorff space with $H^{2}(X, \mathbb{Z})$ countable and $\tau: G \rightarrow$ Aut $C_{0}(X) \cong$ Homeo $(X)$ a continuous action of a second-countable locally compact group on $C_{0}(X)$. Then for every class $[\omega] \in H^{2}(G, C(X, \mathbb{T}))$, there is an action

$$
\alpha: G \rightarrow \text { Aut } A \text {, }
$$

where $A=C_{0}(X) \otimes \mathscr{K}$, differing from $\tau \otimes$ id bv a cocycle with values in Inn $A$, and such that $c(\alpha)=[\omega]$.

Proof. Suppose $G$ is infinite and take $\mathscr{K}=\mathscr{K}\left(L^{2}(G)\right)$, where we use right Haar measure. (If $G$ is finite, first construct $\alpha$ on a suitable $C_{0}(X) \otimes M_{n}$ and then stabilize.) Choose a Borel cocycle $\omega \in Z^{2}(G, C(X, \mathbb{\nabla}))$ and for $x \in X, t \in G$, define $u_{t}(x) \in$ $U\left(L^{2}(G)\right)$ by

$$
\left(u_{t}(x) \xi\right)(s)=\overline{\omega(s, t)(s \cdot x)} \xi(s t), \quad \xi \in L^{2}(G),
$$

Then $u$ is Borel in both variables and

$$
\begin{aligned}
\left(u_{r t}(x)\right)(\xi)(s) & =\overline{\omega(s, r t)(s \cdot x)} \xi(s r t) \\
& =\omega(r, t)(x) \overline{\omega(s, r)(s \cdot x)} \overline{\omega(s r, t)(s \cdot x)} \xi(s r t) \\
& =\omega(r, t)(x) u_{r}(x)\left[u_{t}\left(r^{-1} x\right) \xi\right](s), \quad \text { (by the cocycle identity) }
\end{aligned}
$$

so that $t \mapsto \operatorname{Ad} u_{t}$ is a Borel 1-cocycle $G \rightarrow \operatorname{Inn} A$ for the action given by $\tau \otimes$ id. Thus Ad $u_{t}$ is automatically continuous in $t$ and we may define $\alpha: G \rightarrow$ Aut $A$ by

$$
\begin{aligned}
\left(\alpha_{t} f\right)(x) & =\operatorname{Ad} u_{t}(x) f\left(t^{-1} x\right) \\
& =\left[\left(\operatorname{Ad} u_{t}\right)(\tau \otimes \mathrm{id})_{t}(f)\right](x) .
\end{aligned}
$$


Then by construction, $\alpha$ is a continuous homomorphism from $G$ to Aut $A$, and $c(\alpha)=[\omega]$ since

$$
u_{r t}(x)=\omega(r, t)(x) u_{r}(x) u_{t}\left(r^{-1} x\right)
$$

Our second realization theorem, which is slightly more convenient for our main applications, refers specifically to the case where $G$ splits as a product $G=S \times K$, with $S$ abelian and acting trivially on $X$.

Proposition 3.2. Let $G=S \times K$ be a second-countable locally compact group which splits as a direct product, with $S$ abelian. Let $q: E \rightarrow X$ be a principal $\hat{S}$-bundle (with $E$ and $X$ second-countable and locally compact), and assume $E$ is equipped with an action of $K$ that commutes with the action of $\hat{S}$ (and so induces an action of $K$ on $X$ ). Then there is an action $\alpha$ of $G$ on $A=C_{0}(X, \mathscr{K})$ such that $\left.\alpha\right|_{s}$ is locally unitary, and such that there is an isomorphism of principal $\hat{S}$-bundles $\left(A \rtimes_{\alpha} S\right) \cong E$ intertwining the actions of $K=G / S$.

Proof. Once again, we assume $S$ is infinite; it is easy to make modifications to cover the case of a finite group. Since $\hat{S}$ acts freely and properly on $E$, we know by [Gr1, corollary 15] that $B=C_{0}(E) \rtimes \hat{S}$ is $C_{0}(X)$-isomorphic to $A$. The corresponding homeomorphism of $X=E / \hat{S}$ onto $\hat{B}$ is given by

$$
\psi: G \cdot \xi \mapsto \pi_{\xi}=\operatorname{Ind}_{\{e\}}^{\hat{s}} \operatorname{eval}_{\xi}, \quad \xi \in E,
$$

(cf. [PR2, lemma 3.3]). Since we were given commuting actions of $\hat{S}$ and $K$ on $E$, we obtain an action of $K$ on $B$ commuting with the dual action of $S$, and thus an action $\alpha$ of $G$ on $B$ (or on $A$ ). By [PR2, theorem 3.1], $\left.\alpha\right|_{S}$ is locally unitary, and $\left(B \rtimes_{\alpha} S\right)^{\wedge} \rightarrow X$ is $\hat{S}$-equivariantly isomorphic to $q: E \rightarrow X$, via the map

$$
\varphi: \xi \mapsto \pi_{\xi} \times U, \quad \xi \in E,
$$

where $U$ is the obvious representation of $S$. A simple calculation shows that

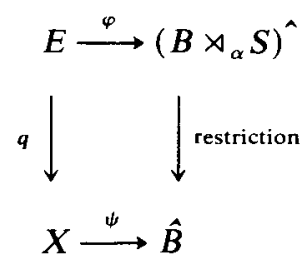

commutes and intertwines the actions of $K$ on $E$ and on $\left(B \rtimes_{\alpha} S\right)^{\hat{n}}$. Using a $C_{0}(X)$-isomorphism $B \cong C_{0}(X, \mathscr{K})$, we may now transport everything to $A$.

Remark 3.3. Despite the great differences in appearance between propositions 3.1 and 3.2, many cases of the latter may be deduced from the former. For example, suppose in the situation of proposition (3.2) that $H^{2}(X, \mathbb{Z})$ is countable and $S$ is connected. By [R, theorem 2.15], the pointwise trivial part of $H^{2}(S, C(X, \mathbb{T}))$ is naturally isomorphic to $H^{1}(X, \hat{\mathbf{S}})$, which classifies principal $\hat{S}$-bundles over $X$ - here $\hat{\mathbf{S}}$ is the sheaf of germs of continuous $\hat{S}$-valued functions. This isomorphism is such that if $\alpha$ is a locally unitary action of $S$ on $A=C_{0}(X, \mathscr{K})$, then the class which we called $c(\alpha) \in H^{2}(X, C(X, \mathbb{T}))$ maps to the class of the $\hat{S}$-bundle $\left(A \rtimes_{\alpha} S\right)^{\wedge} \rightarrow X$. 
Suppose now that we are given $q: E \rightarrow X$ as in proposition (3.2), that $H^{2}(X, \mathbb{Z})$ is countable, and that $q$ is a trivial bundle. Then the action of $K$ on $E \cong X \times \hat{S}$ must be of the form

$$
k \cdot(x, \gamma)=(k \cdot x, \varphi(k, x)+\gamma)
$$

where

$$
\varphi \in Z^{1}(K, C(X, \hat{S}))=Z^{1}(K, \operatorname{Hom}(S, C(X, \pi)))
$$

But

$$
\begin{aligned}
H^{1}(K, \operatorname{Hom}(S, C(X, \mathbb{T}))) & =H^{1}\left(K, H^{1}(S, C(X, \mathbb{T}))\right) \\
& \hookrightarrow H^{2}(K \times S, C(X, \mathbb{T}))
\end{aligned}
$$

via the spectral sequence of [M, theorem 9] (which collapses since we are dealing with a direct product). It is easy to see that if one applies proposition 3.1 to the image of $[\varphi]$ in $H^{2}(K \times S, C(X, \mathbb{T}))$, then the effect will be to prove proposition 3.2 in this case.

Similarly, if $S$ is compact, so that $\varphi$ is a covering map (though not necessarily trivial), and if $K$ and $X$ are connected, then an action of $K$ on $X$ will have at most one lifting to an action of $K$ on $E$. Thus if one applies proposition 3.1 to any class in $H^{2}(S \times K, C(X, \pi))$ which restricts in the pointwise trivial part of $H^{2}(S, C(X, \mathbb{J})) \cong H^{1}(X, \hat{S})$ to the class of $q$, the effect will be to produce an action of $G=S \times K$ on $A$ such that $\left.\alpha\right|_{S}$ is locally unitary and $\left(A \rtimes_{\alpha} S\right)^{\wedge} \cong E$ (as a $K$-space, since $E$ can have only one $K$-action consistent with the $G$-action on $X$ ). Hence we have proved (3.2) for this case as well.

\section{Application of the theory to some specific situations}

Once again we consider the situation of $\S 2$. That is, $A$ is a separable continuous-trace algebra with spectrum $X$, and $\alpha$ is an action of a second-countable locally compact abelian group $G$ on $A$, with the isotropy groups $G_{x}=H(x \in X)$ and the Mackey obstructions in $H^{2}(H, \mathbb{T}$ ) independent of $x$. (Recall we are mostly interested in the case where $A$ is $G$-simple, in which case this is automatic.) $S$ denotes the smallest closed subgroup of $H$ such that the Mackey cocycle is trivial on $S$ and totally skew on $H / S$. We wish to relate the Connes spectrum $\Gamma(\alpha)$ to the structure of $A \rtimes_{\alpha} G$.

Case I: $S$ discrete. In [Goot1, pp. 317-318], an argument was sketched to show that if $S$ is discrete and $A$ is $G$-simple, then $A \rtimes_{\alpha} G$ is simple if and only if $\Gamma(\alpha)=\hat{G}$. We shall now rederive this result from the theory of $\S 2$.

THEOREM 4.1. With notation as above, suppose $A$ is $(G, \alpha)$-simple and $S$ is discrete. Then $\Gamma(\alpha)=\tilde{\Gamma}(\alpha)=\tilde{\Gamma}(\alpha)$ is a closed subgroup of $\hat{G}$ containing $S^{\perp}$, and $\operatorname{Prim}\left(A \rtimes_{\alpha} G\right) \cong \hat{G} / \Gamma(\alpha)$. In particular, $A \rtimes_{\alpha} G$ is simple if and only if $\Gamma(\alpha)=\hat{G}$.

Proof. If $A$ is $(G, \alpha)$-simple, then $A \rtimes_{\alpha} G$ is $(\hat{G}, \hat{\alpha})$-simple [P1, proposition 7.9.6], and in particular, $\hat{G}$ acts minimally on Prim $\left(A \rtimes_{\alpha} G\right) \cong \mathscr{2}\left(\left(A \rtimes_{\alpha} S\right)^{\wedge} / G\right)$ (by proposition 1.2). Since $S^{\perp}$ acts trivially on $(A \rtimes S)^{\wedge}$ and hence acts trivially on Prim $\left(A \rtimes_{\alpha} G\right)$, we have a minimal action of the compact group $\hat{S}$ on Prim $\left(A \rtimes_{\alpha} G\right)$. By the proof of [Gr2, lemma 22], this can only happen if Prim $\left(A \rtimes_{\alpha} G\right)$ is Hausdorff 
and $\hat{S}$ acts transitively. Then from the characterizations of $\Gamma(\alpha), \tilde{\Gamma}(\alpha)$, and $\tilde{\tilde{\Gamma}}(\alpha)$ in proposition 2.2, all three spectra coincide with the inverse image in $\hat{G}$ of the subgroup of $\hat{S}$ that acts trivially on Prim $\left(A \rtimes_{\alpha} G\right)$.

This proves the theorem. One might note as a variant of the proof that $\Gamma(\alpha)=\tilde{\Gamma}(\alpha)$ that $\tilde{\Gamma}(\alpha)$ is always a closed subsemigroup of $\Gamma(\alpha)$, but that in this case, $\tilde{\Gamma}(\alpha)$ is the inverse image in $\hat{G}$ of a closed subsemigroup of $\hat{S}$. Since $\hat{S}$ is compact, $\tilde{\Gamma}(\alpha)$ is then a group $[\mathbf{W r}]$, and it's easy to conclude that $\Gamma(\alpha)=\tilde{\Gamma}(\alpha)$.

Case II: $G$ compact. If $G$ is compact and $A$ is liminary, then the Mackey machine for computing $\left(A \rtimes_{\alpha} G\right)^{\wedge}$ only involves cocycle duals of compact groups, so the crossed product is necessarily liminary. Then $A \rtimes_{\alpha} G$ is of course only simple when $G$ acts transitively on $\hat{A}$ and $S$ is trivial, but nevertheless the topology of $\left(A \rtimes_{\alpha} G\right)^{\wedge}$ is still of interest and sometimes rather complicated if we drop $G$-simplicity of $A$ (cf. [RR, § 2]). Therefore it's interesting to note the following:

Proposition 4.2. Suppose $A$ is a separable continuous-trace algebra and $\alpha: G \rightarrow$ Aut $A$ is a (strongly continuous) action of a compact metrizable abelian group $G$, with constant isotropy group $H$ (for the action of $G$ on $\hat{A}$ ) and with constant symmetrizer group $S$. Then $\Gamma(\alpha)=\tilde{\Gamma}(\alpha)=\tilde{\tilde{\Gamma}}(\alpha)=S^{\perp}, \quad\left(A \rtimes_{\alpha} G\right)^{\wedge} \quad$ is Hausdorff and $\left(A \rtimes_{\alpha} G\right)^{\wedge} \cong$ $\left(A \rtimes_{\alpha} S\right)^{\wedge} / G$. (Note $\left(A \rtimes_{\alpha} S\right)^{\wedge}$ is a covering space of $\hat{A}$ and $G / H$ acts freely on $(A \rtimes S)^{\wedge}$ in a way commuting with the action of $\hat{S}$ by covering transformations.)

Proof. Since $S$ is compact and $\left.\alpha\right|_{S}$ is pointwise unitary, $\left.\alpha\right|_{S}$ is locally unitary by [R, corollary 2.2]. Thus $(A \rtimes S) \hat{\wedge} \rightarrow \hat{A}$ is a locally trivial principal $\hat{S}$-bundle, i.e. a covering projection with covering group $\hat{S}$, since $\hat{S}$ is discrete. In particular, $(A \rtimes S)^{\hat{N}} \cong$ $(A \rtimes H)^{\wedge}$ is Hausdorff, and since $G$ is compact, so is $\mathscr{Q}((A \rtimes S) \hat{H} G)=(A \rtimes S)^{\wedge} / G$. Since $\hat{S} \times(G / H)$ acts freely on $(A \rtimes S) \hat{,} \hat{S}$ acts freely on $(A \rtimes G) \hat{\text {, }}$, hence from the characterization of the various spectra in terms of their action on $(A \rtimes G) \hat{\text {, we have }}$

$$
\Gamma(\alpha)=\tilde{\Gamma}(\alpha)=\tilde{\tilde{\Gamma}}(\alpha)=S^{\perp}
$$

Case III: $S$ compact, $G$ non-compact.

Proposition 4.3. Suppose $A$ is a separable continuous-trace algebra with $\hat{A}=X$ and $\alpha: G \rightarrow$ Aut $A$ is an action of a second-countable locally compact abelian group $G$ on $A$ such that $A$ is $G$-simple. If $S$ and $H$ are as above, $S$ is compact, if $\left.\alpha\right|_{s}$ is unitary (this is automatic if $\left.\stackrel{\tilde{H}}{1}^{1}(X, \hat{S})=0\right)$, and if $G / H$ is connected, then Prim $\left(A \rtimes_{\alpha} G\right) \cong \hat{S}$, and $\Gamma(\alpha)=\tilde{\Gamma}(\alpha)=\tilde{\Gamma}(\alpha)=S^{\perp}$. In particular, $A \rtimes_{\alpha} G$ is simple if and only if $S=\{1\}$ (i.e. $\Gamma(\alpha)=\hat{G}$ ).

Proof. If $G / H$ is connected and $A$ is $G$-simple, then $X$ carries a minimal action of a connected group and so is connected. Since $\hat{S}$ is discrete and $\left(A \rtimes_{\alpha} S\right)^{\wedge} \cong X \times \hat{S}$ if $\left.\alpha\right|_{S}$ is unitary, each 'sheet' $X \times\{\gamma\}$ of $(A \rtimes S) \hat{~}(\gamma \in \hat{S})$ must be $G / H$-invariant and $(G / H)$-minimal, hence

$$
\operatorname{Prim}\left(A \rtimes_{\alpha} G\right) \cong \mathscr{Q}((A \rtimes S) \hat{\wedge} / G) \cong \hat{S}
$$

The last statement follows as in the proof of proposition 4.2

Now we shall see that the conclusion of proposition 4.3 fails in general if $\left.\alpha\right|_{s}$ is only locally unitary but not unitary (and all other hypotheses are the same). 
THEOREM 4.4. Let $X$ be the unit tangent bundle of a compact Riemann surface $M$ of genus $\geq 2$ (i.e. $X=\operatorname{PSL}(2, \mathbb{R}) / \pi$ for $\pi$ some discrete, torsion-free, cocompact subgroup of $\operatorname{PSL}(2, \mathbb{R})$ ). Then there are (many) actions $\alpha$ of $G=\mathbb{R} \times \mathbb{T}$ on $A=C(X) \otimes \mathscr{K}$, such that $\mathbb{T}$ acts trivially on $X$ and $\mathbb{R}$ acts freely and minimally, and such that $A \rtimes_{\alpha} G$ is primitive but not simple. (In other words, $\Gamma(\alpha)=\hat{G}$ but $\tilde{\Gamma}(\alpha) \neq \Gamma(\alpha)$.)

Proof. Let $p: \tilde{M} \rightarrow M$ be any infinite cyclic cover of $M$ such that $\tilde{M}$ is connected. (Since the abelianization of $\pi=\pi_{1}(M)$ is free abelian of rank $\geq 4$, one may obtain many such $p$ 's by taking various surjections $\pi \rightarrow \mathbb{Z}$.) Let $\tilde{X}$ be the unit tangent bundle of the non-compact Riemann surface $\tilde{M}$. By proposition 3.2 (or by remark 3.3 and proposition 3.1), we may choose the action $\alpha$ of $G$ on $A$ so that $\mathbb{T}$ acts trivially on $X,\left(A \rtimes_{\alpha} \mathbb{T}\right)^{\wedge} \rightarrow \hat{A}$ agrees as a $\mathbb{Z}$-covering with the projection $q: \tilde{X} \rightarrow X$, and so that $\mathbb{R}$ acts on $X$ and $\tilde{X}$ by the respective horocycle flows. (If we identify $X$ with $\operatorname{PSL}(2, \mathbb{R}) / \pi$, this means $\mathbb{R}$ acts by left translation by the lower-triangular subgroup, and similarly for $\tilde{X}=\operatorname{PSL}(2, \mathbb{R}) / \tilde{\pi}$.) Then

$$
\operatorname{Prim}\left(A \rtimes_{\alpha} G\right) \cong \mathscr{Q}(\tilde{X} / \mathbb{R}) \text {. }
$$

It is well-known (cf. [Eb2, theorem 4.5]) that the horocycle flow on $X$ is minimal. By [Eb1, theorem 3.8], the geodesic flow on $\tilde{X}$ is topologically transitive, hence by [Eb2, theorem 3.3], the non-wandering set of the horocycle flow on $\tilde{X}$ is all of $\tilde{X}$. Then by [Eb2, theorem 4.1], the horocycle flow on $\tilde{X}$ has a dense orbit, i.e. $A \rtimes_{\alpha} G$ is primitive. On the other hand, since $\tilde{M}$ is non-compact, the horocycle flow on $\tilde{X}$ cannot be minimal, by [Eb2, theorem 4.5]. Note that the analysis in [Eb2] could be used to obtain a more detailed picture of Prim $\left(A \rtimes_{\alpha} G\right)$ - this space is very complicated.

Note that in this argument, we may replace $\mathbb{R} \times \mathbb{T}$ by $\mathbb{R} \times \mathbb{J}^{n}$ and $\mathbb{Z}$ by $\mathbb{Z}^{n}$ (for any $n$ ), provided that we take the genus of $M$ to be $\geq n / 2$.

Case IV: $S$ non-compact, non-discrete. When $S$ is non-compact and non-discrete, even when $H=S=\mathbb{R}, G=\mathbb{R}^{2}$, and $\left.\alpha\right|_{S}$ is unitary, it can happen that $A \rtimes_{\alpha} G$ is primitive but not simple. Thus the results above (especially theorem 4.1) are in a sense sharp.

THEOREM 4.5. There are actions $\alpha$ of $G=\mathbb{R} \times \mathbb{R}$ on $A=C(X) \otimes \mathscr{K}$, with $X$ a compact metric space, with $\left.\alpha\right|_{\mathbb{R} \times\{0\}}$ unitary, and with $\{0\} \times \mathbb{R}$ acting freely and minimally on $X$, such that $A \rtimes_{\alpha} G$ is primitive but not simple, i.e. $\Gamma(\alpha)=\hat{G}$ but $\tilde{\Gamma}(\alpha) \neq \Gamma(\alpha)$. One may even choose $X$ to be a compact manifold and the action of $G$ on $X$ to be smooth.

Proof. We take $X$ to be a compact metric space admitting a free minimal $\mathbb{R}$-action which is not uniquely ergodic. For an example of such a space, note that M. Herman constructs in $[\mathbf{H}, \S 6]$ a minimal, non-uniquely ergodic diffeomorphism of $\mathbb{I}^{2}$, and one may suspend to get a flow on a compact 3-manifold with the right properties. By theorem 4.8 below, there is a continuous 1-cocycle

$$
\varphi \in Z^{1}(\mathbb{R}, C(X, \mathbb{R}))
$$

for this flow, such that if we take the corresponding skew-product flow

$$
s \cdot(x, t)=(s \cdot x, \varphi(s, x)+t), \quad s, t \in \mathbb{R}, x \in X,
$$


we obtain an action of $\mathbb{R}$ on $X \times \mathbb{R}$ which has a dense orbit but is not minimal, and which projects to the original $\mathbb{R}$-action on $X$. By our realization theorems, proposition 3.2 or proposition 3.1 and remark 3.3, we may now construct an action $\alpha$ of $\mathbb{R}^{2}$ on $C(X) \otimes \mathscr{K}$ with the desired properties.

To complete the proof of theorem 4.5, we need a construction of continuous cocycles for a flow which is not uniquely ergodic. This is done in theorem 4.8 , which first requires two simple lemmas. The first is classical and may be found in [KB, theorem II, p. 93].

LеммА 4.6. Let $X$ be a compact metric space equipped with an action of $\mathbb{R}$, and let $\mu$ be an ergodic invariant probability measure. Then there is an $\mathbb{R}$-invariant Borel set $E \subseteq X$ such that $\mu(E)=1$ and such that for any $f \in C(X)$ and $x \in E$,

$$
t^{-1} \int_{0}^{t} f(s \cdot x) d s \rightarrow \int f d \mu .
$$

Proof. Choose a countable dense subset $\left\{f_{i}\right\}$ of $C(X)$. For each $i$, the Birkhoff ergodic theorem asserts that there is a $\mu$-null set $N_{i}$ such that

$$
t^{-1} \int_{0}^{t} f_{i}(s \cdot x) d s \rightarrow \int f d \mu
$$

for $x \notin N_{i}$. Then clearly $E=X \backslash \bigcup_{i} N_{i}$ has the desired property.

LEMMA 4.7. (a) Let $Y$ be a second-countable locally compact space, and let $\nu_{0}, \nu_{1}$, and $\nu_{2}$ be probability measures supported on disjoint Borel sets $C_{0}, C_{1}$ and $C_{2}$. (In other words, $C_{i} \cap C_{j}=\varnothing$ for $i \neq j$ and $\nu_{j}\left(C_{k}\right)=\delta_{j k}$.) Then $\nu_{2}$, viewed as a linear functional on

$$
V=\left\{f \in C_{0}(Y): \int f d \nu_{0}=\int f d \nu_{1}=0\right\},
$$

has norm 1.

(b) Let $Y$ be as in (a) and let $\mu_{0}, \mu_{1}$ be probability measures supported on disjoint Borel sets. Then $\mu_{1}$, viewed as a linear functional on

$$
\left\{f \in C_{0}(Y): \int f d \mu_{0}=0\right\}
$$

has norm 1.

Proof. (a) Since $\nu_{2}$ is a probability measure, clearly $\left\|\left.\nu_{2}\right|_{v}\right\| \leq 1$. We need to prove the reverse inequality. Since $\nu_{0}$ and $\nu_{1}$ are linearly independent, we may choose $g_{0}$, $g_{1} \in C_{0}^{\mathbb{R}}(Y)$ with

$$
\int g_{j} d \nu_{k}=\delta_{j k} ; \quad j, k=0,1 .
$$

Let $C=\max \left(\left\|g_{0}\right\|,\left\|g_{1}\right\|\right)$ and let $\varepsilon>0$. For any small $\delta>0$, we may by the density of $C_{0}^{\mathbb{R}}(Y)$ in $L^{1}$ choose $g \in C_{0}^{\mathbb{R}}(Y)$ with $\|g\| \leq 1$ such that

$$
\int\left|g-\chi_{C_{2}}\right| d\left(\nu_{0}+\nu_{1}+\nu_{2}\right) \leq \delta .
$$


Then in particular,

$$
\int g d \nu_{2} \geq 1-\delta, \quad \int g d \nu_{0} \leq \delta, \quad \int g d \nu_{1} \leq \delta
$$

Let

$$
h=g-\left(\int g d \nu_{0}\right) g_{0}-\left(\int g d \nu_{1}\right) g_{1} .
$$

Then $h \in V,\|h\| \leq 1+2 C \delta$, and $\int h d \nu_{2} \geq 1-\delta-2 C \delta$, so

$$
\left\|\left.\nu_{2}\right|_{v}\right\| \geq(1-(2 C+1) \delta)(1+2 C \delta)^{-1} \text {, }
$$

which will be $\geq 1-\varepsilon$ for a suitable choice of $\delta$.

(b) This follows immediately from (a), applied to the disjoint union of $Y$ with a point, and with $\nu_{2}=\mu_{1}, \nu_{0}=\mu_{0}, \nu_{1}=$ point measure at the added point.

THEOREM 4.8. Let $X$ be a compact metric space with a free minimal $\mathbb{R}$-action which is not uniquely ergodic. Then there exists $\psi \in C^{\mathbb{R}}(X)$ such that for the action of $\mathbb{R}$ on $X \times \mathbb{R}$ given by

$$
t(x, r)=\left(t \cdot x, r+\int_{0}^{t} \psi(s \cdot x) d s\right)
$$

there is at least one dense orbit, but not all orbits are dense.

Proof. Let $\mu_{0}$ and $\mu_{1}$ be distinct ergodic probability measures for the $\mathbb{R}$-action on $X$, and use lemma 4.6 to find corresponding (necessarily disjoint) Borel sets $E_{0}, E_{1}$ with

$$
\begin{gathered}
\mu_{j}\left(E_{k}\right)=\delta_{j k} ; \quad j, k=0,1, \\
t^{-1} \int_{0}^{t} f(s \cdot x) d s \rightarrow \int f d \mu_{j} \quad \text { for } f \in C(X), \quad x \in E_{j} ; \quad j=0,1 .
\end{gathered}
$$

Fix a point $x_{0} \in E_{0}$.

We shall construct $\psi \in C^{\mathbb{R}}(X)$ with $\|\psi\| \leq 1$ so that the $\mathbb{R}$-orbit of $\left(x_{0}, 0\right)$ is dense in $X \times \mathbb{R}$ for the action based on the cocycle constructed out of $\psi$. We will construct $\psi$ as $\psi=\sum_{n=0}^{\infty} \psi_{n}$, where $\psi_{n}$ 's are constructed inductively to satisfy

$$
\left\{\begin{array}{l}
\left\|\psi_{n}\right\| \leq 2^{-n-1}, \\
\int \psi_{n} d \mu_{0}=0 \quad \text { for all } n, \\
\int \psi_{0} d \mu_{1}=\frac{1}{4}, \quad \int \psi_{n} d \mu_{1}=0 \text { for } n \geq 1,
\end{array}\right.
$$

as well as additional conditions $(* * *)$ to be specified shortly.

Choose a sequence $\left\{z_{i}\right\}$ dense in $X$ and a sequence $\left\{r_{i}\right\}$ dense in $\mathbb{R}$ so that $\left|r_{i}\right| \leq i$ and

$$
\left\{\left(z_{i}, r_{i}\right)\right\} \text { is dense in } X \times \mathbb{R} .
$$

We shall choose another sequence $\left\{t_{i}\right\}$ in $\mathbb{R}$ inductively; begin by setting $t_{0}=0$. Start the induction by choosing any $\psi_{0}$ satisfying $(*)$, i.e. with

$$
\left\|\psi_{0}\right\| \leq 2^{-1}, \quad \int \psi_{0} d \mu_{0}=0, \quad \int \psi_{0} d \mu_{1}=2^{-2}
$$


(This is possible by lemma 4.7(b) applied with $X$ in place of $Y$, which asserts that $\mu_{1}$ restricted to $\left\{f \in C(X): \int f d \mu_{0}=0\right\}$ has norm 1.) Suppose $n \geq 1$ and $t_{j}, \psi_{j}$ have been chosen for $j<n$. Choose $t_{n} \in \mathbb{R}$ with

$$
\left\{\begin{array}{l}
t_{n}>2 t_{n-1}, \quad t_{n}>n \cdot 2^{n+3}, \\
\operatorname{dist}\left(t_{n} \cdot x_{0}, z_{n}\right)<2^{-n} \\
\left|\frac{1}{t_{n}} \int_{0}^{t_{n}}\left(\psi_{0}+\psi_{1}+\cdots+\psi_{n-1}\right)\left(s \cdot x_{0}\right) d s\right|<2^{-n-3} .
\end{array}\right.
$$

This is possible by the defining property of $E_{0}$ (recall $x_{0} \in E_{0}$ ) together with minimality of the flow on $X$. Next, choose $\psi_{n} \in C(X)$ satisfying $(*)$, i.e. with

$$
\left\|\psi_{n}\right\| \leq 2^{-n-1}, \quad \int \psi_{n} d \mu_{0}=\int \psi_{n} d \mu_{1}=0
$$

as well as the conditions

$(* * *) \quad\left\{\begin{array}{l}\psi_{n}\left(s \cdot x_{0}\right)=0 \text { for } 0 \leq s \leq t_{n-1}, \\ \frac{1}{t_{n}} \int_{0}^{t_{n}} \psi_{n}\left(s \cdot x_{0}\right) d s=\frac{r_{n}}{t_{n}}-\frac{1}{t_{n}} \int_{0}^{t_{n}}\left(\psi_{0}+\cdots+\psi_{n-1}\right)\left(s \cdot x_{0}\right) d s .\end{array}\right.$

To see that this is possible, we apply lemma $4.7(\mathrm{a})$ with

$$
\left\{\begin{array}{l}
Y=X \backslash\left\{s \cdot x_{0}: 0 \leq s \leq t_{n-1}\right\}, \quad \nu_{0}=\mu_{0}, \quad \nu_{1}=\mu_{1}, \\
\int f d \nu_{2}=\frac{1}{t_{n}-t_{n-1}} \int_{t_{n-1}}^{t_{n}} f\left(s \cdot x_{0}\right) d s, \text { and } \\
C_{0}=E_{0} \backslash\left\{s \cdot x_{0}: 0 \leq s \leq t_{n}\right\}, \quad C_{1}=E_{1}, \quad C_{2}=\left\{s \cdot x_{0}: t_{n-1} \leq s \leq t_{n}\right\} .
\end{array}\right.
$$

Thus $\nu_{2}$ has norm 1 on $\operatorname{ker} \mu_{0} \cap \operatorname{ker} \mu_{1} \cap C_{0}(Y)$. Now

$$
\left|\frac{r_{n}}{t_{n}}-\frac{1}{t_{n}} \int_{0}^{t_{n}}\left(\psi_{0}+\cdots+\psi_{n-1}\right)\left(s \cdot x_{0}\right) d s\right| \leq \frac{n}{t_{n}}+2^{-n-3}<2^{-n-2},
$$

while for $f \in C_{0}(Y)$, extended to be 0 on $\left\{s \cdot x_{0}: 0 \leq s \leq t_{n-1}\right\}$,

$$
\frac{1}{t_{n}} \int_{0}^{t_{n}} f\left(s \cdot x_{0}\right) d s=\frac{t_{n}-t_{n-1}}{t_{n}} \int f d \nu_{2} .
$$

Since $\left(t_{n}-t_{n-1}\right) / t_{n}>\frac{1}{2}$, we conclude that we can choose $\psi_{n} \in \operatorname{ker} \mu_{0} \cap C_{0}(Y)$ satisfying $\left\|\psi_{n}\right\| \leq 2^{-n-1}$ and $(* * *)$. This completes the inductive step.

Now let $\psi=\sum_{n=1}^{\infty} \psi_{n}$. By construction,

and

$$
\|\psi\| \leq 1, \quad \int \psi d \mu_{0}=0, \quad \int \psi d \mu_{1}=\frac{1}{4},
$$

$$
\begin{aligned}
\int_{0}^{t_{n}} \psi\left(s \cdot x_{0}\right) d s & =\int_{0}^{t_{n}}\left(\psi_{0}+\cdots+\psi_{n}\right)\left(s \cdot x_{0}\right) d s+\int_{0}^{i_{n}} \sum_{j>n} \psi_{j}\left(s \cdot x_{0}\right) d s \\
& =r_{n}+0=r_{n} .
\end{aligned}
$$

Thus

$$
\begin{aligned}
\operatorname{dist}\left(\left(t_{n} \cdot x_{0}, \int_{0}^{t_{n}} \psi\left(s \cdot x_{0}\right) d s\right),\left(z_{n}, r_{n}\right)\right) & =\operatorname{dist}\left(\left(t_{n} \cdot x_{0}, r_{n}\right),\left(z_{n}, r_{n}\right)\right) \\
& =\operatorname{dist}\left(t_{n} \cdot x_{0}, z_{n}\right)<2^{-n},
\end{aligned}
$$

so the $\mathbb{R}$-orbit of $\left(x_{0}, 0\right)$ is dense in $X \times \mathbb{R}$. 
On the other hand, if $x_{1} \in E_{1}$, then the $\mathbb{R}$-orbit of $\left(x_{1}, 0\right)$ is not dense, because

$$
t^{-1} \int_{0}^{t} \psi\left(s \cdot x_{1}\right) d s \rightarrow \int \psi d \mu_{1}=\frac{1}{4}
$$

so that

$$
\int_{0}^{t} \psi\left(s \cdot x_{1}\right) d s>t / 8
$$

for large $t$, and thus the closure of the orbit of $\left(x_{1}, 0\right)$ in $X \times \mathbb{R}$ cannot include all of $X \times\{0\}$. This completes the proof of the theorem.

Acknowledgements. We would like to thank the Mathematical Sciences Research Institute for the hospitality and for the congenial atmosphere that made this collaboration possible. We would also like to thank Marina Ratner for drawing our attention to [Eb2].

This project and all authors were partially supported by NSF Grant 8120790 , and the authors were partially supported individually: S. Hurder by NSF Grant MCS8201604; D. Olesen by the Danish Natural Science Research Council; I. Raeburn by the Australian Research Grants Scheme; J. Rosenberg by NSF Grant DMS8400900 .

\section{REFERENCES}

[AM] L. Auslander \& C. C. Moore. Unitary Representations of Solvable Lie Groups. Mem. Amer. Math. Soc., no. 62 (1966).

[BK] L. Baggett \& A. Kleppner. Multiplier representations of abelian groups. J. Funct. Anal. 14 (1973), 299-324.

[Br] O. Bratteli. Crossed products of UHF algebras by product type actions. Duke. Math. J. 46 (1979), 1-23.

[Eb1] P. Eberlein. Geodesic flow in certain manifolds without conjugate points. Trans. Amer. Math. Soc. 167 (1972), 151-170.

[Eb2] P. Eberlein. Horocycle flows on certain surfaces without conjugate points. Trans. Amer. Math. Soc. 233 (1977), 1-36.

[ET] D. E. Evans \& H. Takai. Simplicity of crossed products of GCR algebras by abelian groups. Math. Ann. 243 (1979), 55-62.

[Goot1] E. C. Gootman. On certain properties of crossed products. Proc. Symp. Pure Math. (Amer. Math. Soc.) 38 (1982), Part I, 311-322.

[Goot2] E. C. Gootman. Abelian group actions on Type I $C^{*}$-algebras. Preprint, University of Georgia.

[GO] E. C. Gootman \& D. Olesen. Spectra of actions on type I $C^{*}$-algebras. Math. Scand. 47 (1980), 329-349.

[GR] E. C. Gootman \& J. Rosenberg. The structure of crossed product $C^{*}$-algebras: a proof of the generalized Effros-Hahn conjecture. Invent. Math. 52 (1979), 283-298.

[Gr1] P. Green. $C^{*}$-algebras of transformation groups with smooth orbit space. Pacific J. Math. 72 (1977), 71-97.

[Gr2] P. Green. The local structure of twisted covariance algebras. Acta Math. 140 (1978), 191-250.

[H] M. R. Herman. Construction d'un difféomorphisme minimale d'entropie topologique non nulle. Ergod. The. Dynam. Sys. 1 (1981), 65-76.

[Ki] A. Kishimoto. Simple crossed products of $C^{*}$-algebras by locally compact abelian groups. Yokohama Math. J. 38 (1980), 69-85.

[KB] N. Kryloff \& N. Bogoliouboff. La théorie générale de la mesure dans son application à l'étude des systèmes dynamiques de la méchanique nonlinéare. Annals of Math. 38 (1937), 65-113. 
[La] E. C. Lance. Automorphisms of postliminal $C^{*}$-algebras. Pacific J. Math. 23 (1967), 547-555.

[LOP] M. B. Landstad, D. Olesen \& G. K. Pedersen. Towards a Galois theory for $C^{*}$-crossed products. Math. Scand. 43 (1978), 311-321.

[M] C. C. Moore. Group extensions and cohomology for locally compact groups, III, Trans. Amer. Math. Soc. 221 (1976), 1-33.

[O1] D. Olesen. A classification of ideals in crossed products. Math. Scand. 45 (1979), 157-167.

[OP1] D. Olesen \& G. K. Pedersen. Applications of the Connes spectrum to $C^{*}$-dynamical systems. J. Funct. Anal. 30 (1978), 179-197.

[OP2] D. Olesen \& G. K. Pedersen. Applications of the Connes spectrum to $C^{*}$-dynamical systems, II, J. Funct. Anal. 36 (1980), 18-32.

[OP3] D. Olesen \& G. K. Pedersen. Applications of the Connes spectrum to $C^{*}$-dynamical systems, III. J. Funct. Anal. 45 (1982), 357-390.

[P1] G. K. Pedersen. $C^{*}$-algebras and their Automorphism Groups. London Math. Soc. Monographs Vol. 14. Academic Press, London, 1979.

[P2] G. K. Pedersen. Dynamical systems and crossed products. Proc. Symp. Pure Math. (Amer. Math. Soc.) 38 (1982), Part I, 271-283.

[PR1] J. Phillips \& I. Raeburn. Automorphisms of $C^{*}$-algebras and second Čech cohomology. Indiana Univ. Math. J. 29 (1980), 799-822.

[PR2] J. Phillips \& I. Raeburn. Crossed products by locally unitary automorphism groups and principal bundles. J. Operator Th. 11 (1984), 215-241.

[RR] I. Raeburn \& J. Rosenberg. Crossed products of continuous-trace $C^{*}$-algebras by smooth actions. Preprint MSRI, 1985, No. 05711-85.

[R] J. Rosenberg. Some results on cohomology with Borel cochains, with applications to group actions on operator algebras. Preprint MSRI, 1985, No. 03611-85.

[Wi] D. P. Williams. The topology on the primitive ideal space of transformation group $C^{*}$. algebras and $C C R$ transformation group $C^{*}$-algebras. Trans. Amer. Math. Soc. 266 (1981), 335-359.

[Wr] F. B. Wright. Semigroups in compact groups. Proc. Amer. Math. Soc. 7 (1956), 309-311. 